\title{
NGST Advanced Cryocooler Technology Development Program (ACTDP) Cooler System
}

\author{
D. Durand, R. Colbert, C. Jaco, M. Michaelian, \\ T. Nguyen, M. Petach, and E. Tward \\ Northrop Grumman Space Technology (NGST) \\ Redondo Beach, California, 90278 USA
}

\begin{abstract}
The Northrop Grumman Space Technology (NGST) Advanced Cryocooler Technology Development Program (ACTDP) cooler features a 5-6 K Joule-Thomson (J-T) cooler precooled by a three-stage Pulse Tube (PT) cryocooler to provide remote cooling in the range of 60 to $70 \mathrm{~mW}$ at temperatures ranging from 5 to 6 Kelvin. This cooler was developed under contract with the Jet Propulsion Laboratory to enable active cooling of large space telescopes, with particular focus on the Mid Infrared Instrument (MIRI) of the James Webb Space Telescope (JWST). This paper describes recent progress in the design and performance of the NGST ACTDP cooler applied to the specific requirements of the MIRI application. End-to-end performance of the MIRI Cooler Subsystem is projected by combining separate performance measurements of the J-T cooler and the PT precooler using flight like hardware. Significant improvement in the efficiency of NGST's low temperature three-stage pulse tube cryocooler enables our design to meet the nominal steady state MIRI cooling requirements of $65 \mathrm{~mW}$ at $<6.2 \mathrm{~K}$ and $77 \mathrm{~mW}$ total parasitic load on the 18 Kelvin refrigerant lines for a total bus power of $256 \mathrm{~W}$, against the requirement of $360 \mathrm{~W}$ maximum. This paper also addresses the extent to which this design can accommodate changes in the heat load requirements.
\end{abstract}

\section{INTRODUCTION}

The MIRI cooler application features a requirement for remote cooling of the infrared focal plane arrays directly behind the primary optics of JWST, with the vast majority of the cooler mass located on the spacecraft bus, six meters away. Figure 1 indicates the location of the Cooler Compressor Assembly (CCA), containing the Joule-Thomson compressor and precooler recuperators as well as the entire pulse tube cryocooler, and the Cold Head Assembly (CHA), containing the final J-T recuperator and J-T restriction to achieve 6 Kelvin. Not shown in Figure 1, are the Cryocooler Control Electronics (CCE) located elsewhere on the spacecraft bus and the deployable refrigerant lines connecting the CCA to the CHA.

\section{DESIGN}

The design of the MIRI Cooler Subsystem, shown in Figure 2, is based on a 4He Joule- Thomson (J-T) cooler, providing remote 6 Kelvin cooling, and an all Pulse Tube (PT) precooler. ${ }^{1,2}$ The 


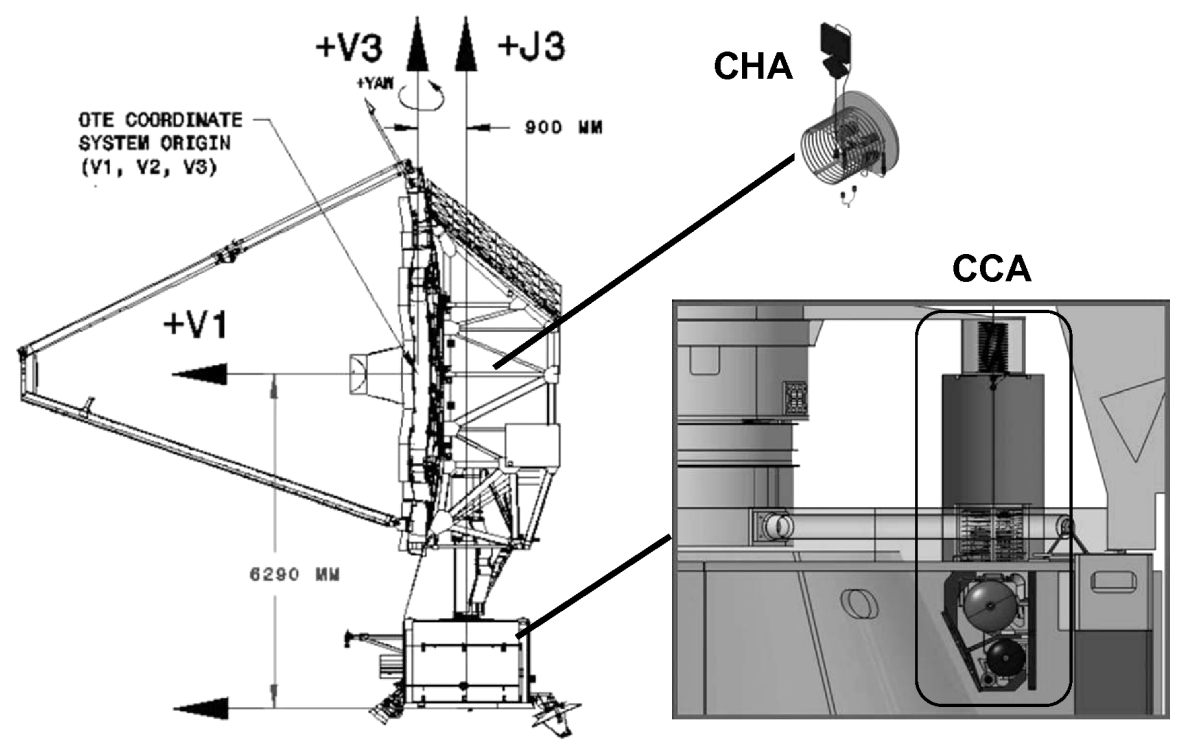

Figure 1. The MIRI Cooler Subsystem. The line drawing on the left, showing the spacecraft bus and the Optical Telescope Element (OTE) of JWST is from the MIRI Cooler Subsystem request for proposal. The Cooler Compressor Assembly (CCA) and the Cold Head Assembly (CHA) are sown as expanded CAD renderings on the right hand side. The CCA is shown in context of the spacecraft bus and tower structures in the immediate vicinity. The Cryocooler Control Electronics (CCE) and the Cooler Tower Assembly (CTA) are not shown.

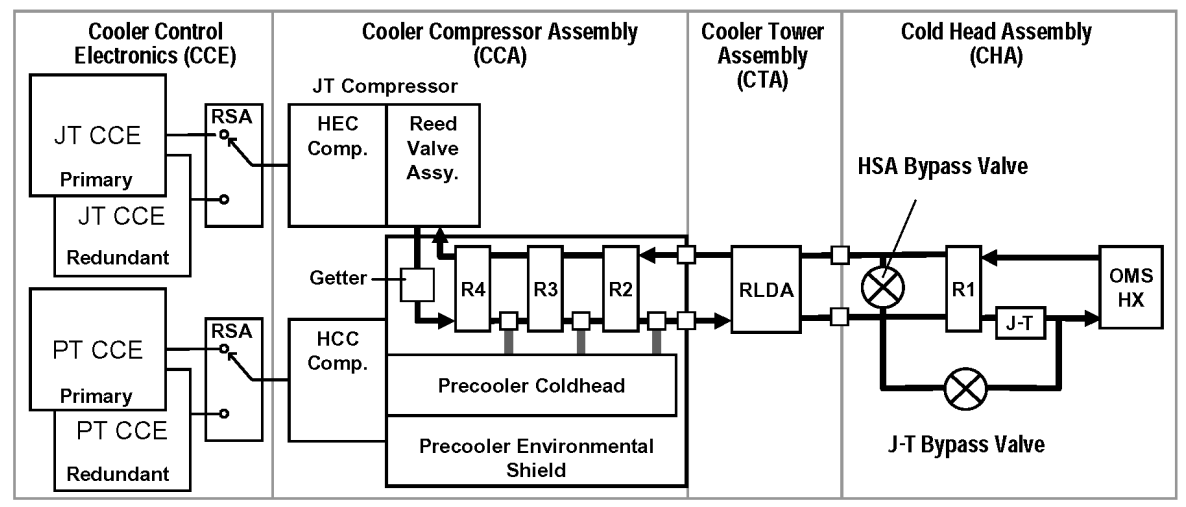

Figure 2. Block diagram of the ACTDP design applied to the MIRI Cooler Subsystem. The dark lines show the He gas flow in the J-T cooler loop.

J-T and PT compressors in the MIRI Cooler are each driven by a standby redundant pair of CCE units of the Advanced Cryocooler Electronics (ACE) design. Relay Switch Assemblies (RSA) connect the redundant pair of CCEs to each compressor. The pulse tube precooler consists of a High Capacity Cryocooler (HCC) compressor and a three-stage pulse tube cold head. The design of the precooler is very similar to the design of NGST's 10 Kelvin pulse tube cryocooler, described elsewhere in this proceedings. ${ }^{3}$ The Joule-Thomson cooler is based on a valved version of NGST's High Efficiency Cryocooler (HEC) compressor. A reed valve assembly converts the oscillating pressure wave produced by the HEC compressor into a continuous flow of He gas around the J-T loop. Helium gas passes through recuperators R4, R3, and R2 with three heat exchangers connected to the three stages of the PT cold head as shown in Figure 2. The net result is efficient precooling of 
the He gas to approximately 18 Kelvin. Refrigerant lines carry the precooled He gas from the Cooler Compressor Assembly (CCA), located on the spacecraft bus, to the Cold Head Assembly (CHA), located at MIRI.

The refrigerant lines between the CCA and the CHA are coiled to form the Refrigerant Line Deployment Assembly (RLDA), enabling the deployment of the tower between the spacecraft bus and the telescope element. The drop in temperature from 18 Kelvin to less than 6 Kelvin is accomplished by the recuperator, R1, and the J-T restriction, shown as "J-T" in the block diagram. A normally closed bypass valve is used during cool down from 100 Kelvin to $\sim 15$ Kelvin. In this range of temperature the valve is opened to allow He gas to bypass the recuperator and the J-T restriction. Once the OMS has been cooled to $\sim 15$ Kelvin, the J-T bypass valve is closed causing gas to flow through the restriction and recuperator in the cold head assembly. After passing through the so-called "pinch point" the cooler subsystem reaches steady state operation with the OMS heat exchanger somewhat below 6 Kelvin.

In addition to the explicit 6 Kelvin heat load at the OMS heat exchanger, the MIRI cooler design must account for a large parasitic heat load on the RLDA at 18 Kelvin. The ACTDP cooler design allowed for the option of cooling an additional explicit heat load at 18 Kelvin to actively intercept the conductive parasitic heat load from the MIRI electrical harness. Intercepting heat from the harness in this way is feasible but was selected against on a cost-benefit basis due to added complexity in the harness routing and interface definition.] The steady state performance of the MIRI Cooler Subsystem can be characterized by the total input power required as a function of the explicit heat load at the 6 Kelvin heat exchanger and the parasitic load on the 18 Kelvin refrigerant lines, dominated by the load on the RLDA.

\section{PERFORMANCE}

Bus power is calculated using an end-to-end J-T loop thermal performance model. Measurements of the CCE efficiency, the J-T compressor efficiency, the performance characteristics of the $\mathrm{J}$-T recuperators, heat exchangers and restriction, and the efficiency of the pulse tube cryocooler, are used to anchor the model. Recent improvements in our low temperature three-stage pulse tube cryocooler have significantly reduced the input power required for the MIRI Cooler Subsystem. This improved overall performance is shown in Figure 3. The contours of constant total bus power indicate the extent to which the MIRI Cooler design can accommodate changes in the heat load requirements. For example, if the 6 Kelvin load remained $65 \mathrm{~mW}$, but the 18 Kelvin load increased to

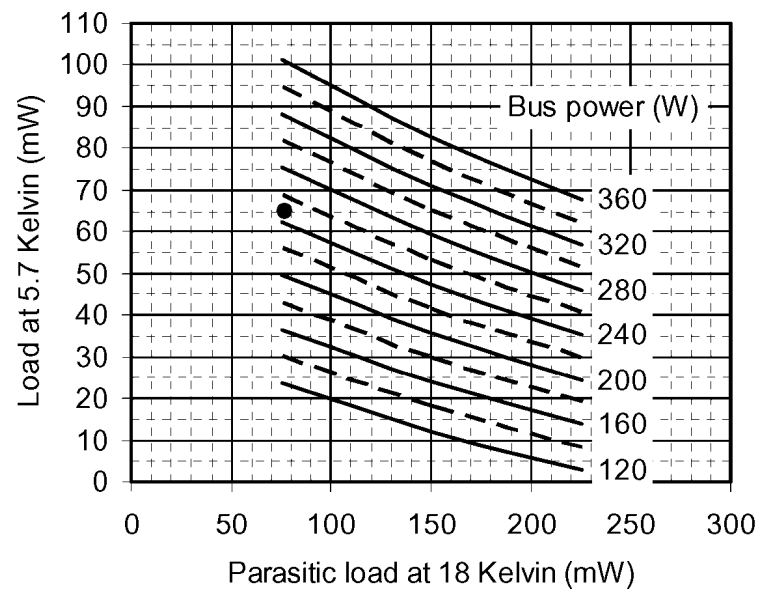

Figure 3. Total bus power as a function of the explicit heat load at 5.7 Kelvin and the parasitic heat load at 18 Kelvin. Contours of constant total bus power ranging from $120 \mathrm{~W}$ to $360 \mathrm{~W}$ are shown (see labels). The solid point marks the nominal MIRI Cooler Subsystem heat loads. 


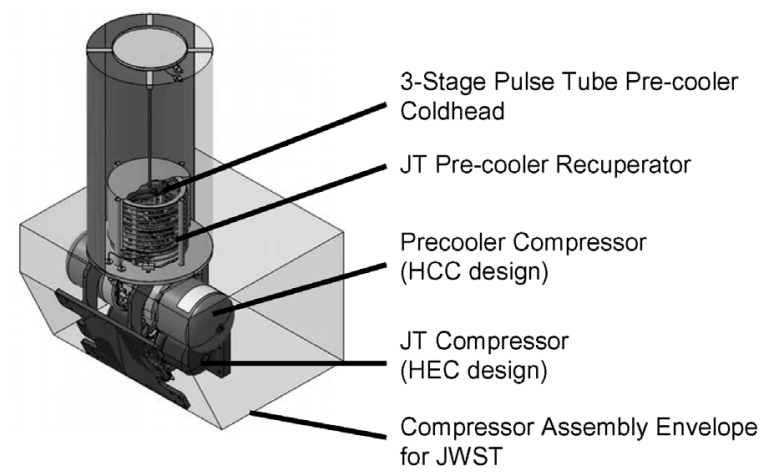

Figure 4. Mechanical layout of the Cooler Compressor Assembly (CCA).

$150 \mathrm{~mW}$, the total bus power for steady state operation would be $300 \mathrm{~W}$ (see Figure 3). The model also calculates the total power required to transverse the pinch point in each case.

\section{MECHANICAL DESIGN}

The mechanical layout of Northrop Grumman's design for the MIRI Cooler Subsystem CCA is shown in Figure 4. The CCA hardware is shown in relation to the envelope as specified in the MIRI Cooler Subsystem Specification from the Jet Propulsion Laboratory. Compressors for the pulse tube and J-T coolers are located as indicated. The recuperators R2, R3, and R4 are physically sections along the length of the coil labeled "J-T Precooler Recuperator" in Figure 4. The pulse tube cryocooler cold head is located inside the recuperator coil.

A technical challenge identified early on is the design trade between structural strength and low conducted parasitic heat load in the PT precooler cold head/J-T precooler recuperator structure. Our approach has been to maintain the three-stage pulse tube cold head and the recuperator and support as independent structures, connected by highly compliant thermal straps.

Techniques for designing the pulse tube cold head to survive launch vibration are based on our standard practices for a variety of linear and coaxial cold heads. The recuperator and support structure is relatively unique to the MIRI cooler design, so component-level launch vibration testing to verify the approach is warranted.

Figure 5 shows the recuperator launch vibration test model in test at an NGST launch vibration test facility. Finite element analysis was used to assess the robustness to the launch vibration envi-

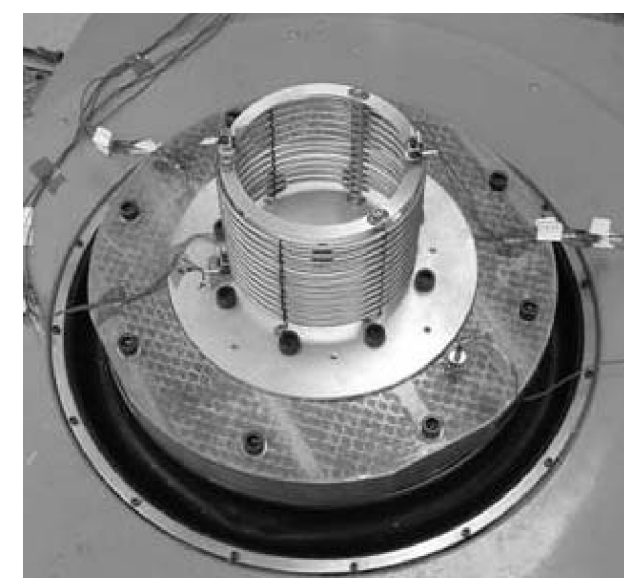

Figure 5. Recuperator launch vibration test. The recuperator model tested is structurally accurate. 
ronment of the design prior to the test. Once verified in simulation the test model recuperator structure was tested in excess of the MIRI cooler qualification level of 14.4 grms.

Structural integrity was verified by comparing the resonant frequencies and amplitudes with the simulations and between before and after tests. The structure passed this component-level launch vibration testing.

\section{CONCLUSIONS}

The MIRI Cooler Subsystem design has been shown to have good margin against the specifications and have the required structural strength in component and subassembly-level tests. The next step in the development is demonstrating performance of the end-to-end MIRI cooler and robustness to launch vibration of an integrated CCA.

\section{ACKNOWLEDGMENTS}

The authors gratefully acknowledge continued technical discussions with the JPL cryocooler group, led by Ron Ross Jr., and the JPL MIRI cryocooler team, led by Melora Larson. This work was carried out by Northrop Grumman Space Technology and sponsored by the Jet Propulsion Laboratory Advanced Cryocooler Technology Development Program.

\section{REFERENCES}

1. D. Durand, J. Raab, R. Colbert, M. Michaelian, T. Nguyen, M. Petach, and E. Tward, "NGST Advanced Cryocooler Technology Development Program (ACTDP) Cooler System," Adv. In Cryogenic Engin., Vol 51A, Amer. Inst. of Physics, New York (2006), pp. 615-622.

2. Raab, J., Colbert, R., Godden, J., Harvey, D., Michaelian, M., Nguyen, T., Petach, M., Orsini, R., Tward, E., and Waterman, M., "NGST Advanced Cryocooler Technology Development Program (ACTDP) Cooler System," Cryocoolers 13, Edited by Ross, R.G., Jr., Springer Science+Business Media, New York (2005), pp. 9-14.

3. Nguyen, T., Colbert, R., Durand, D., Jaco, C., Michaelian, M., Tward. E., "10K Pulse Tube Cooler," Cryocoolers 14, ICC Press, Boulder, CO (2007), this proceedings. 